The Astrophysical Journal, 608:L109-L112, 2004 June 20

(C) 2004. The American Astronomical Society. All rights reserved. Printed in U.S.A.

\title{
MID-INFRARED OBSERVATIONS OF VAN MAANEN 2: NO SUBSTELLAR COMPANION
}

\author{
J. FARIHI AND E. E. BECKLIN \\ Department of Physics and Astronomy, University of California at Los Angeles, 8371 Math Sciences Building, \\ Los Angeles, CA 90095-1562; jfarihi@astro.ucla.edu, becklin@ astro.ucla.edu \\ AND \\ B. A. Macintosh \\ Institute of Geophysics and Planetary Physics, Lawrence Livermore National Laboratory, \\ 7000 East Avenue L-413, Livermore, CA 94551; bmac@igpp.1lnl.gov \\ Received 2004 March 23; accepted 2004 May 12; published 2004 May 18
}

\begin{abstract}
The results of a comprehensive infrared imaging search for the putative $0.06 M_{\odot}$ astrometric companion to the $4.4 \mathrm{pc}$ white dwarf van Mannen 2 are reported. Adaptive optics images acquired at $3.8 \mu \mathrm{m}$ reveal a diffractionlimited core of 0.09 and no direct evidence of a secondary. Models predict that at $5 \mathrm{Gyr}$, a $50 M_{\mathrm{J}}$ brown dwarf would be only 1 mag fainter than van Maanen 2 at this wavelength, and the astrometric analysis suggested a separation of 0"2. In the case of a chance alignment along the line of sight, a 0.4 mag excess should be measured. An independent photometric observation at the same wavelength reveals no excess. In addition, there exist published Infrared Space Observatory observations of van Maanen 2 at 6.8 and $15.0 \mu \mathrm{m}$ that are consistent with the photospheric flux of a $6750 \mathrm{~K}$ white dwarf. If recent brown dwarf models are correct, there is no substellar companion with $T_{\text {eff }} \gtrsim 500 \mathrm{~K}$.
\end{abstract}

Subject headings: binaries: general — stars: individual (van Maanen 2) — stars: low-mass, brown dwarfs — white dwarfs

\section{INTRODUCTION}

Van Maanen's star, also known as van Maanen 2, was discovered in 1917 by Adrian van Maanen (van Maanen 1917). Its discovery was quite accidental, as van Maanen was searching for common proper-motion companions to HD 4628 (Lalande 1299) and noticed the even larger proper motion of a nearby star. At 4.4 pc, van Maanen 2 is the nearest single white dwarf (Holberg et al. 2002).

Makarov (2004) reported the astrometric detection of a substellar companion to van Maanen 2 through analysis of Hipparcos data. The data suggested an orbital solution with a lower bound companion mass of $0.06 \pm 0.02 M_{\odot}$, a period of $1.57 \mathrm{yr}$, and a maximum separation on the sky of 0 "'3. Based on the published parameters, a binary orbital calculator (A. Ghez 2004, private communication) indicated a separation of 0.19 at position angle $274^{\circ}$ near the middle of 2004 January. This Letter presents the results of a direct imaging search carried out during that time frame.

\section{OBSERVATIONS, DATA REDUCTION, AND PHOTOMETRY}

\subsection{Keck Adaptive Optics Observations}

Van Maanen 2 was observed using the facility adaptive optics system (Wizinowich et al. 2000a, 2000b) and the NIRC2 camera at Keck Observatory on 2004 January 12. Five dithered images were obtained through an $L^{\prime}(3.4-4.1 \mu \mathrm{m})$ filter. To reduce thermal backgrounds and provide a more symmetric point-spread function (PSF), a circular pupil stop was used that describes an inscribed circle on the hexagonal Keck primary, with a diameter of $\sim 9 \mathrm{~m}$. Each image consisted of 100 co-adds of $0.4 \mathrm{~s}$ each. Van Maanen 2 itself was used as the guide star, with the adaptive optics system running at a rate of $80 \mathrm{~Hz}$. The correction was quite good, with a Strehl ratio (measured relative to the PSF of the $9 \mathrm{~m}$ pupil) of 0.75 and an FWHM of 0".089, essentially diffraction-limited. The plate scale was 0 ."01 pixel $^{-1}$.

The adaptive optics images were reduced using standard programs in the IRAF environment. For a given raw image, the sky was extracted by taking the median of the four remaining dithered images with rejection of hot and cold pixels. A flat frame was created by averaging all five sky frames and subsequently normalizing. Each dithered frame was then skysubtracted and flat-fielded, and the resulting five frames were registered and averaged.

Owing to the uncertainties in the wings of the adaptive optics PSF and weather that was not photometric, it was difficult to perform photometry on the Keck data. Therefore, photometric observations of van Maanen 2 were acquired at the Infrared Telescope Facility (IRTF).

\subsection{IRTF and ISO Observations}

The $3.8 \mu \mathrm{m}$ data on van Maanen 2 and two standard stars were acquired on 2004 February 1 by A. Stockton at the $3 \mathrm{~m}$ NASA IRTF with NSFCAM (Rayner et al. 1993). For the standards, a nine-point dither pattern was used with each frame consisting of $0.15 \mathrm{~s}$ exposure times 100 co-adds-yielding a total integration time of $135 \mathrm{~s}$. The UKIRT faint standard SA 92-342 ( $\left.L^{\prime}=10.44 \mathrm{mag}\right)$ was observed immediately before van Maanen 2, and the Elias standard star HD $22686\left(L^{\prime}=\right.$ $7.19 \mathrm{mag}$ ) was observed immediately afterward (Leggett et al. 2003). Two sets of images, acquired in the same manner as the standards, were obtained for van Maanen 2 for a total of 18 frames and $270 \mathrm{~s}$ integration. Conditions during the observations were reported as photometric.

The data on the two standards were reduced using standard programs in the IRAF environment. For a given raw image, the sky was extracted by taking the median of the eight remaining dithered images with rejection of hot and cold pixels. 
TABLE 1

Photometric Data on van MaAnen 2

\begin{tabular}{cccc}
\hline \hline Filter & $\begin{array}{c}\lambda_{0} \\
(\mu \mathrm{m})\end{array}$ & Magnitude & \multicolumn{1}{c}{$\begin{array}{c}F_{\nu} \\
(\mathrm{mJy})\end{array}$} \\
\hline$B \ldots \ldots \ldots \ldots$ & 0.44 & 12.91 & $27.9 \pm 1.4$ \\
$V \ldots \ldots \ldots \ldots$ & 0.55 & 12.39 & $40.2 \pm 1.2$ \\
$R \ldots \ldots \ldots \ldots$ & 0.64 & 12.13 & $43.1 \pm 1.3$ \\
$I \ldots \ldots \ldots \ldots$ & 0.80 & 11.90 & $42.0 \pm 1.3$ \\
$J \ldots \ldots \ldots \ldots$ & 1.22 & 11.69 & $34.4 \pm 0.6$ \\
$H \ldots \ldots \ldots$ & 1.65 & 11.57 & $24.7 \pm 0.5$ \\
$K_{s} \ldots \ldots \ldots$ & 2.16 & 11.50 & $16.7 \pm 0.5$ \\
$L^{\prime} \ldots \ldots \ldots$ & 3.76 & 11.40 & $6.8 \pm 0.4$ \\
$\mathrm{LW} 2 \ldots \ldots$ & 6.75 & $\ldots$ & $2.0 \pm 0.4$ \\
$\mathrm{LW} 3 \ldots \ldots$ & 15.0 & $\ldots$ & $0.5 \pm 0.2$ \\
\hline
\end{tabular}

NoTES. $-B V R I$ photometry is from Bergeron et al. (2001) and is on the Johnson-Cousins system. $J H K_{s}$ data are from 2MASS and agree with the near-infrared data reported by Bergeron et al. (2001). The $L^{\prime}$ data are from the present work, and the LW2 and LW3 fluxes are from ISO (Chary et al. 1999).

A flat frame was created by averaging all nine sky frames and subsequently normalizing. Each dithered frame was then skysubtracted and flat-fielded, and the resulting nine frames were registered and averaged. Reducing the two sets of images on van Maanen 2 was more difficult owing to the faintness of the target in each raw frame. It was found that the target was more easily seen in pairwise-subtracted images rather than in skysubtracted images. All 18 pairwise-subtracted frames were flatfielded, registered, and averaged, creating one final image for photometric measurements.

The flux of both standards was measured with aperture radii of 1".2 and 2".4 and corrected for extinction. The error in determining the zero point was $0.03 \mathrm{mag}$. Van Maanen 2 was measured in the smaller aperture to minimize noise, corrected to the larger aperture (with an error of $0.04 \mathrm{mag}$ ), and extinctioncorrected. This yielded a signal-to-noise ratio of 48 and a measurement error of $0.03 \mathrm{mag}$. The photometric measurement including all errors was $L^{\prime}=11.40 \pm 0.06$ mag for van Maanen 2.

ISOCAM observations of van Maanen 2 were carried out in 1997 in an effort to provide observational constraints on the origin of metals in the photospheres of cool white dwarfs (Chary et al. 1999). Data were taken at 6.8 and $15.0 \mu \mathrm{m}$ on van Maanen 2 and several other white dwarfs as well as an A0 V calibrator star. The data are listed in Table 1.

\section{RESULTS AND DISCUSSION}

\subsection{Adaptive Optics: No Direct Evidence}

In order to estimate the brightness of the reported companion, a model for a $50 M_{\mathrm{J}}$ brown dwarf at $5 \mathrm{Gyr}$ was chosen. This mass lies conservatively in the lower range of possible values and required no interpolation within available models. The model age is likely to be greater than the $3.67 \mathrm{Gyr}$ cooling age (Bergeron et al. 2001) of van Maanen 2 plus the $\sim 0.5 \mathrm{Gyr}$ main-sequence lifetime (Maeder 1990) for an $\sim 4 M_{\odot}$ progenitor of the $0.83 M_{\odot}$ white dwarf (Weidemann 1987, 1990, 2000; Bragaglia et al. 1995; Bergeron et al. 2001). A substellar companion of this mass and age would be a late $\mathrm{T}$ dwarf $\left(T_{\text {eff }} \sim\right.$ $800 \mathrm{~K}$ ) and have $M_{L^{\prime}}=14.3 \mathrm{mag}$, which is $L^{\prime}=12.5 \mathrm{mag}$ at $4.4 \mathrm{pc}$ (Burrows et al. 1997). Photometric $L^{\prime}$-band data on known brown dwarfs do exist, and the measurements agree with the models used here to within 0.3 mag for spectral type T6 (Leggett et al. 2002). Van Maanen 2 is predicted to have

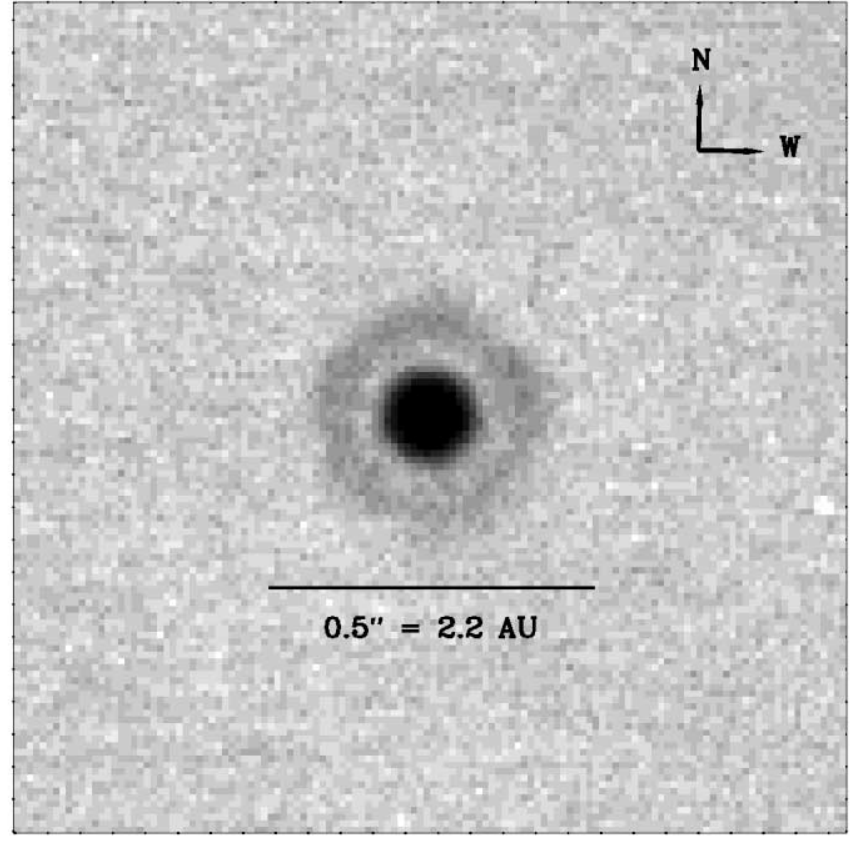

FIG. 1.-The $3.8 \mu \mathrm{m}$ adaptive optics image of van Maanen 2. There is no direct evidence for a companion 1 mag fainter than the primary.

$L^{\prime}=11.43 \mathrm{mag}$ based on the model-predicted $V-K=0.84$ color of a $6750 \mathrm{~K}$ helium white dwarf (Bergeron et al. 1995) and the $K-L^{\prime}=0.12$ color of a $6750 \mathrm{~K}$ blackbody; $V-K=$ 0.89 is the measured color-the extrapolation was done from $V$ in case of any contamination by a companion at $K$.

The reduced $L^{\prime}$ image (Fig. 1) shows no indication whatsoever of a companion with the brightness expected from a brown dwarf of the type reported by Makarov (2004). From the published orbital parameters, the companion should have been at a separation of $00^{\prime \prime} 19$ and a position angle of $274^{\circ}$ on the date of the observation. The FWHM of van Maanen 2 in the reduced image is 0.089 , and the distance to the first Airy ring is $\approx 0^{\prime \prime} .14$. There are two extremely faint features within the Airy ring at position angles of $284^{\circ}$ and $295^{\circ}$. Small aperture flux measurements, relative to the primary, at eight different evenly spaced locations around the Airy ring indicate that these features are unlikely to be real. The flux at $284^{\circ}$ and $295^{\circ}$, $f / f_{0}=0.069$ and 0.073 , respectively, are both within $2 \sigma(0.018)$ of the average flux $\left(f / f_{0}=0.056\right)$ in the ring and are almost certainly artifacts due to imperfect optical guide star corrections. If the feature at $295^{\circ}$ were real, its brightness after subtracting the flux of the primary in the Airy ring implies $L^{\prime}=$ $15.9 \mathrm{mag}$ and a mass of $\sim 15 M_{\mathrm{J}}$, assuming an age of $5 \mathrm{Gyr}$ (Burrows et al. 1997). This is simply not massive enough to cause the reported astrometric wobble.

An artificial star was planted at 0 ". 19 from the primary in order to test the ability to detect faint companions at this separation. The adaptive optics PSF, extracted from the reduced image of van Maanen 2, was used for the stellar profile of the planted star. To be conservative, this artificial star is a full $2.0 \mathrm{mag}$ fainter than the white dwarf- this was confirmed by placing the star at many positions on the image and measuring its flux without contamination by the primary. The simulated star is readily seen in Figure 2.

Large errors in the reported orbital parameters allow for the possibility that the companion remained unresolved owing to chance alignment during the observation. The values and com- 


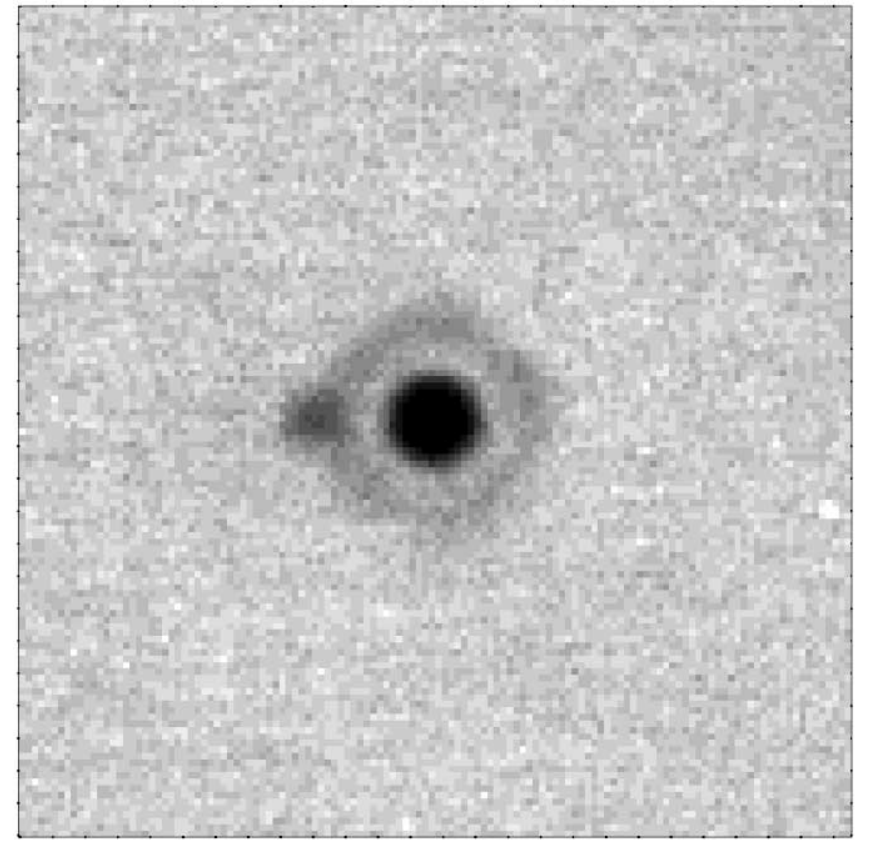

FIG. 2.-Adaptive optics image of van Maanen 2 with an artificial star placed at 0.19 from the white dwarf. The magnitude of the planted star is $L^{\prime} \approx$ $13.4 \mathrm{mag}$ and is clearly seen.

puted errors in separation and position angle, derived from the binary orbital calculator, are $0.19_{-0.18}^{+0.07}$ arcsec and $274_{-2}^{+180} \mathrm{deg}$, respectively. Hence, the putative companion could have been located at almost any position in its orbit by the epoch of the observation. However, there is further evidence against this possibility.

\subsection{Photometry: No Indirect Evidence}

In the case of a chance alignment, any substellar companion would still cause excess emission at mid-infrared wavelengths. This is primarily due to the size difference between brown dwarfs and white dwarfs: a ratio of $10: 1$ in radius. It is not possible for a significant occultation to occur. At most, a white dwarf could block only $\sim 1 \%$ of the light from an orbiting brown dwarf.

As mentioned above, a value of $L^{\prime}=11.40 \pm 0.06$ mag was

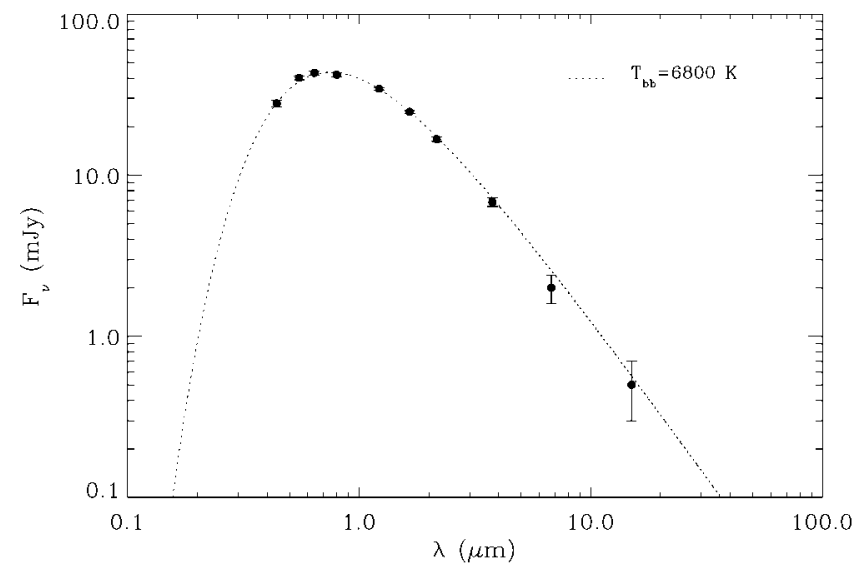

FIG. 3.- Spectral energy distribution of van Maanen 2 from the data in Table 1 . Overplotted is a $6800 \mathrm{~K}$ blackbody demonstrating that the data reveal no excess emission in the infrared and are consistent with a single star. measured for van Maanen 2. This is to be compared with the value predicted for a $6750 \mathrm{~K}$ white dwarf, $L^{\prime}=11.43 \mathrm{mag}$. The combined flux of the white dwarf plus a $50 M_{\mathrm{J}}$ brown dwarf at 5 Gyr would have a magnitude of $L^{\prime}=11.09$ mag. Hence, it is concluded that there is no excess emission at this wavelength.

Furthermore, the published Infrared Space Observatory (ISO) observations place even stronger constraints on the absence of flux from a brown dwarf around van Maanen 2. A recent model (Burrows et al. 2003) of the flux from a $5 \mathrm{Gyr}$, $25 M_{\mathrm{J}}$ brown dwarf was integrated over the $I S O 15.0 \mu \mathrm{m}$ filter LW3. This results in a flux of about $1.0 \mathrm{mJy}$ in this filter. The reported measurement taken at van Maanen 2 in 1997 was $0.5 \pm 0.2 \mathrm{mJy}$. This measurement is consistent with photospheric flux from the white dwarf, and an excess of $5 \sigma$ most likely would have been detected. If the $I S O$ results are correct and the models are right, any companion with $T_{\text {eff }} \gtrsim 500 \mathrm{~K}$ is ruled out - this includes $10 \mathrm{Gyr}$ old brown dwarfs with $M \geq$ $35 M_{\mathrm{J}}$ (Burrows et al. 1997, 2003).

Table 1 summarizes all existing photometric data on van Maanen 2, and the corresponding fluxes are plotted in Figure 3. It should be clear from the figure that the measured fluxes are all consistent with a single white dwarf with a temperature around $6800 \mathrm{~K}$.

\section{CONCLUSION}

Three compelling infrared observations of van Maanen 2 are presented that rule out the presence of a substellar companion warmer than $\sim 500 \mathrm{~K}$. First, $3.8 \mu \mathrm{m}$ adaptive optics images reveal no significant flux at the predicted magnitude and position of the putative companion reported by Makarov (2004). Second, $3.8 \mu \mathrm{m}$ photometric observations are consistent with no excess emission at this wavelength, as would be expected from a $50 M_{\mathrm{J}}$ brown dwarf at 5 Gyr. And third, $15.0 \mu \mathrm{m}$ data also are consistent with no excess emission, ruling out any possible companion massive enough to induce the reported astrometric wobble.

Part of the data presented herein were obtained at Keck Observatory, which is operated as a scientific partnership among the California Institute of Technology (CIT), the University of California, and the National Aeronautics and Space Administration (NASA). This publication makes use of data acquired at IRTF, which is operated by the University of Hawaii under cooperative agreement NCC 5-538 with NASA, Office of Space Science, Planetary Astronomy Program. Some data used in this Letter are part of the Two Micron All Sky Survey (2MASS), a joint project of the University of Massachusetts and the Infrared Processing and Analysis Center (IPAC)/CIT, funded by NASA and the National Science Foundation. The 2MASS data were retrieved from the NASA/IPAC Infrared Science Archive, which is operated by the Jet Propulsion Laboratory, CIT, under contract with NASA. The authors wish to express their gratitude to A. Stockton, J. Rayner, and A. Tokunaga for their assistance in acquiring the IRTF observations of van Maanen 2. Sincere thanks go to J. Larkin and M. Barczys for donating some of their Keck adaptive optics time for this project. Acknowledgement also goes to B. Hansen for bringing this system to our attention, to B. Zuckerman for a careful reading of this manuscript and many discussions, to A. Ghez for the binary orbit calculator, and to B. Vacca for help in the data reduction. Part of this work was performed under the auspices of the US Department of Energy, National Nuclear 
Security Administration, by the University of California, Lawrence Livermore National Laboratory, under contract W-
7405-Eng-48. This research has been supported in part by grants from NASA to UCLA.

\section{REFERENCES}

Bergeron, P., Leggett, S. K., \& Ruiz, M. T. 2001, ApJS, 133, 413

Bergeron, P., Wesemael, F., \& Beauchamp, A. 1995, PASP, 107, 1047

Bragaglia, A., Renzini, A., \& Bergeron, P. 1995, ApJ, 443, 735

Burrows, A., Sudarsky, D., \& Lunine, J. I. 2003, ApJ, 596, 587

Burrows, A., et al. 1997, ApJ, 491, 856

Chary, R., Zuckerman, B., \& Becklin, E. E. 1999, in The Universe as Seen by ISO, ed. P. Cox \& M. F. Kessler (ESA SP-427; Noordwijk: ESA), 289

Holberg, J. B., Oswalt, T. D., \& Sion, E. M. 2002, ApJ, 571, 512

Leggett, S. K., et al. 2002, ApJ, 564, 452 2003, MNRAS, 345,144

Maeder, A. 1990, in Astrophysical Ages and Dating Methods, ed. E. VangioniFlam, J. Audouze, M. Cassé, \& J. Trân Thanh Vân (Gif-sur-Yvette: Editions Frontières), 71
Makarov, V. V. 2004, ApJ, 600, L71

Rayner, J. T., et al. 1993, Proc. SPIE, 1946, 490

van Maanen, A. 1917, PASP, 29, 258

Weidemann, V. 1987, A\&A, 188, 74 1990, ARA\&A, 28, 103 2000, A\&A, 363, 647

Wizinowich, P., Acton, D. S., Lai, O., Gathright, J., Lupton, W., \& Stomski, P. 2000a, Proc. SPIE, 4007, 2

Wizinowich, P., et al. 2000b, PASP, 112, 315 